\title{
APPLICATION OF ADDITIVE TECHNOLOGIES FOR GROWING LARGE PROFILED SINGLE CRYSTALS OF TUNGSTEN AND MOLYBDENUM
}

\author{
V.A. SHAPOVALOV, V.V. YAKUSHA, A.N. GNIZDYLO and Yu.A. NIKITENKO \\ E.O. Paton Electric Welding Institute, NASU \\ 11 Kazimir Malevich Str., 03680, Kiev, Ukraine. E-mail: office@paton.kiev.ua
}

\begin{abstract}
The paper considers application of additive welding technologies with regard to growing the super large single crystals of refractory metals. Main methods of tungsten and molybdenum single crystals production from liquid phase were analyzed. The perspectives of plasma-induction technology for growing the large plane single crystals of tungsten and molybdenum are shown. A process scheme of additive growth of the plane single crystals of refractory metals using plasma-induction method is described. It is determined that the developed method provides for the possibility of flexible regulation of thermal field of single crystal being grown. Application of local molten zone, formed by low power plasma arc with the parameters typical for welding processes, allows growing the tungsten large single crystals of $210 \times 180 \times 20 \mathrm{~mm}$ size. The crystals are formed under conditions of heating with inductor high-frequency field to the temperature typical for hot deformation range. Given are the results of investigation of structural perfection of the growing crystals which verify the fact that the conditions of single crystals formation in plasma-induction zone melting provide for higher quality of single-crystalline structure than the methods, in which additional heating (electron beam and plasma-arc) is not used. It is determined that the plasma-induction process is characterized by formation of the regular dislocation structures, for which dislocation coalescence in low-angel boundaries are typical. 9 Ref., 7 Figures.
\end{abstract}

Keywords: additive welding technologies, plasma-arc zone melting, tungsten and molybdenum single crystals, dislocation substructure

Growing the tungsten and molybdenum single crystals has own peculiarities related with physical properties of these metals, in particular, high melting temperature. Specifically high melting temperature determined two melting methods based on application of highly-concentrated heat sources, i.e. electron beam and plasma arc. The electron beam heating [1] gained the largest application in commercial production of single crystals.

A traditional scheme of tungsten single crystal growing is a non-crucible zone melting or methods of growing with floating zone (Figure 1). The crystals grown using indicated method have perfect structure

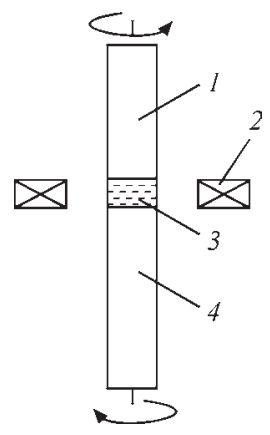

Figure 1. Scheme of vertical non-crucible zone melting: 1 polycrystalline rod; 2 - heater; 3 - «floating» zone; 4 - single crystal and smooth surface, irregularies and roughness of which depend on stability of process modes, namely stability of keeping the power, generated by ring-cathode EB gun, and stability of zone movement. However, at all relative simplicity of the growing method a serious drawback, namely crystal diameter limitation, was found. Increase of diameter rises EB gun power, molten zone volume, overheating temperature and possibility of melt run over. The process became unstable. The crystals produced with such a method had maximum diameter $25 \mathrm{~mm}$ for tungsten and $30 \mathrm{~mm}$ for molybdenum [2].

Found difficulties were partially solved by using a periphery zone melting. But, this method did not allow significant increase of diameter of grown crystals. Besides, diameter increase provoked rise of crystal stresses that resulted in deterioration of single-crystal structure $[3,4]$.

Application of low-temperature plasma and usage of modified Verneuil method (Figure 2) reviled the possibilities of technology development in scope of crystal diameter increase and capability of growing the profiled single crystals.

The crystals grown with the help of plasma arc heating had more rough surface, some geometry deviation was observed (Figure 3), and number of struc- 


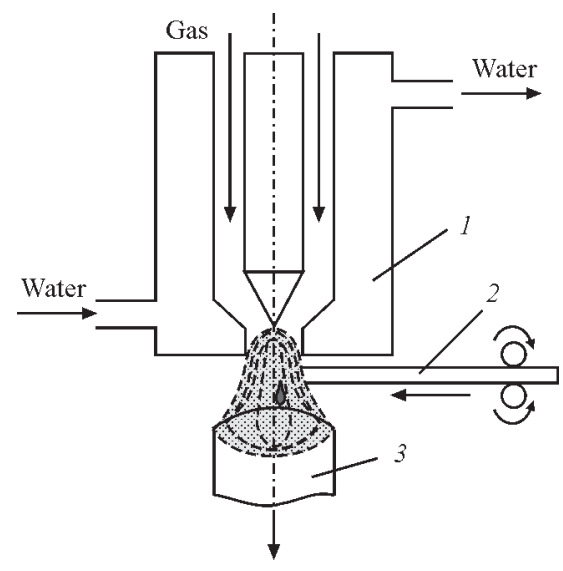

Figure 2. Scheme of growing the single crystals of refractory metals using plasma-arc heating: 1 - plasmatron; 2 - rod; 3 crystal

tural disturbances was an order higher the same index for the crystals grown using EB heating [5].

Further improvement of the technology of tungsten and molybdenum single crystal growing took place due to development at the PWI of a new method for growing the large single crystals by plasma-induction zone melting. The main differences of the method are heating of growing single crystal by high-frequency field energy, preventing the metal pool run over using electro-magnetic forces appearing at interaction of inductor current and current induced in the crystal, and the most important, layer-by-layer (additive) growth of single crystal due to movement of local metal pool, replenished by means of remelting of consumable polycrystalline rod (Figure 4).

Namely application of a local metal pool, formed by a low power plasma arc, with parameters typical for welding processes, allowed developing indicated method for growing super large profiled single crystals of tungsten and molybdenum of $210 \times 180 \times 20 \mathrm{~mm}$ size (Figure 5) [6].

Essence of the method lies in the fact that plasmatron by reciprocating motion moves a metal pool, which is replenished from remelted rods and forms a layer-by-layer crystal, reminding arc surfacing. After each plasmatron pass the single crystal moves downward per deposited layer height, thus, providing stable conditions, in which growing process takes place. The crystal is formed under conditions of heating by

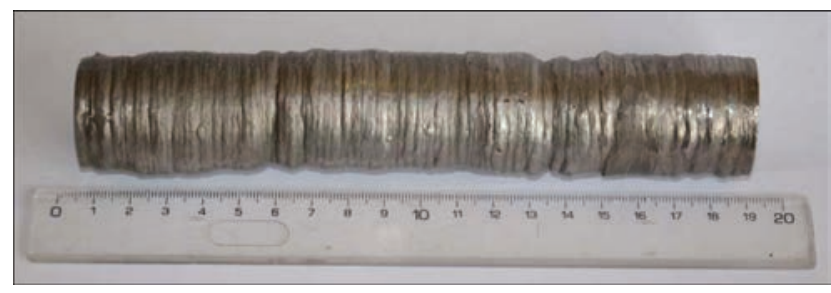

Figure 3. View of tungsten single crystal grown using plasma-arc heating

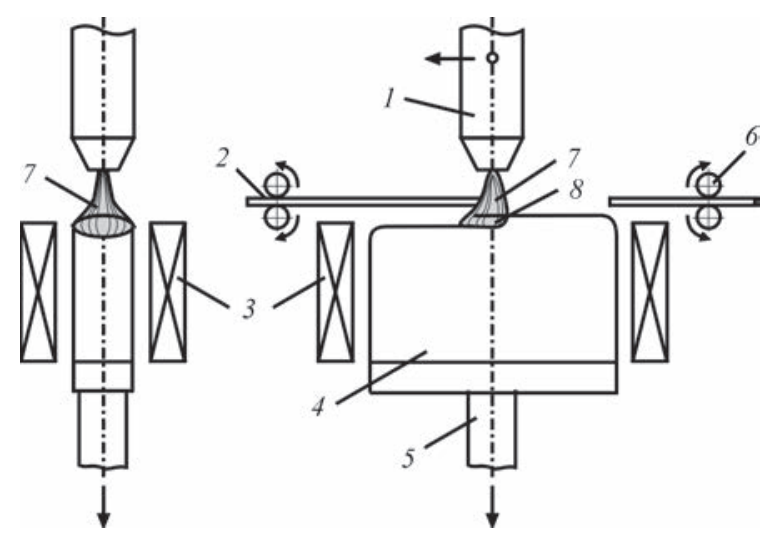

Figure 4. Scheme of unit for additive growing the refractory metal single crystals using plasma-induction method: 1 - plasmatron; 2 - consumable rod; 3 - inductor; 4 - single crystal; 5 - hearth; 6 - rod feeder; 7 - plasma arc

high-frequency inductor field to temperatures typical for hot deformation range.

It is a well-known fact that dislocation displacement takes place at these temperatures under simultaneous effect of external stresses and temperature. The dislocations are not rigidly tightened to «their» slip plane and can migrate from one plane to another, selecting the easiest way. It is considered as additional degree of freedom of dislocation. Such an irregular dislocation movement rises the possibility of their hitting. Therefore, on the one hand, amount of cases of their annihilation (dislocation density decrease) rises, and, on the other hand, there is a tendency to formation of regular dislocation structures, for which coalescence of dislocations in low-angle boundaries is typical (Figure 6). The conditions of single crystal formation provides for higher quality of single-crystal structure than the methods, which do not use additional heating (electron beam and plasma arc) [7].

The crystals grown using indicted method have less smooth side surface, but it does not prevent their application without additional treatment as the billets for large-format rolling.

There are interesting results of crystal structure investigation using optical and X-ray methods. Fig-

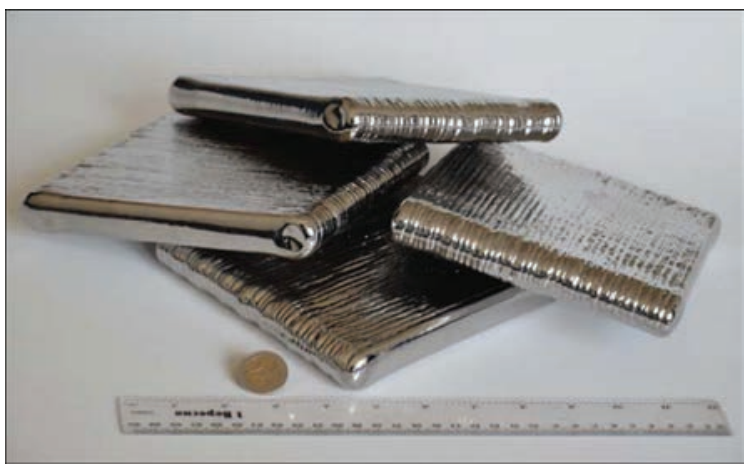

Figure 5. Tungsten single crystals made by plasma-induction method 


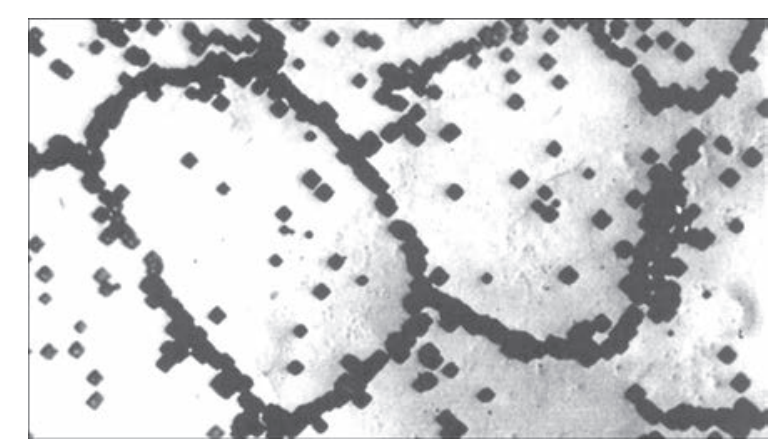

Figure 6. Microstructure $(\times 400)$ of tungsten (flatness $(001))$

ure 7 shows the results of $X$-ray examination of single crystals.

The comparative investigations show that the structure of such crystals is more perfect than plasma-arc one, large macromosaic structure is virtually absent. Further accumulation of experimental facts allows optimizing regulation of structure formation in crystal growing.

Development of additive welding technologies applicable to growth of super large single crystals allows creating new material and expanding it application in the following areas $[8,9]$ :

- X-ray devices (shields, anticathodes);

- electronics (targets for spraying, crucibles for growing oxides and nitrides single crystals);

- electrical engineering (connectors and wires);

- laser equipment (mirrors for optical and X-ray lasers);

- nuclear power engineering (thermionic converters of space power plants, ITER diverters, elements of active part);

- aerospace engineering (nozzle).

\section{Conclusions}

1. Analysis of existing opproaches for producing single crystals allows making a conclusion that the plasma-induction method does not have disadvantages typical for earlier developed methods, and provides for the widest possibilities for process of growing super large profiled tungsten single crystals.

2. Conditions of single crystal formation in plasma-induction zone melting provide for higher quality of single-crystal structure than the methods, in which additional heating is not used. Plasma-induction method is characterized by formation of regular dislo-

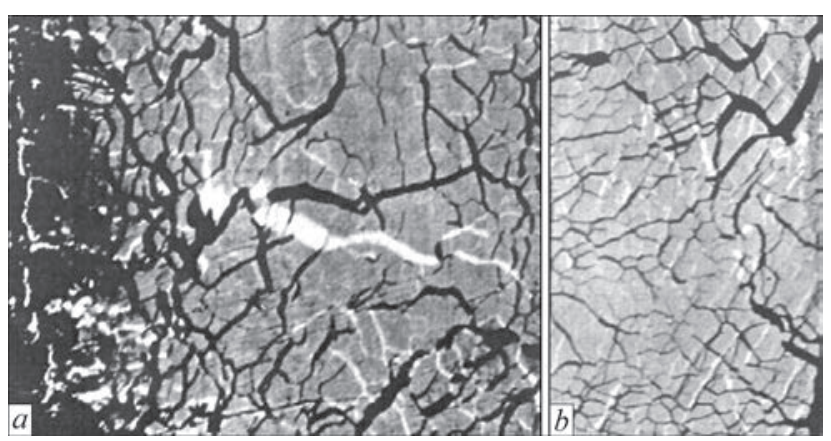

Figure 7. X-ray topogram of angle scanning of reflection: $a-$ longitudinal; $b$ - cross section of crystal

cation structures, for which coalescence in low-angel boundaries is mainly typical.

3. The most perspective directions in application of large oriented single crystals are heat and X-ray shields, ITER diverters, shells of emitters of thermionic converters of nuclear power units, mirrors of power lasers etc.

1. Manokhin, A.I., Burkhanov, G.S. (1987) State-of-the-art of problem of metal single crystals. In: High-frequency and single crystal metallic materials, 5-13. Moscow: Nauka.

2. Kervalishvili, I.D., Shchelkin, Yu.F. (1982) Heat conditions - factor determining the manufacturing of perfect crystals. Fizika i Khimiya Obrab. Materialov, 5, 70-78.

3. Predtechensky, B.S., Starostina, L.S. (1972) Dislocations in molybdenum single crystals produced by zone melting. In: Growth and defecfs of metal crystals, 213-217. Kiev: Naukova Dumka.

4. Glebovsky, V.G., Semenov, V.N., Lomejko, V.V. (1987) Influence of crystallization conditions on structural perfection of tungsten single crystals. In: High-pure and single-crystal metallic materials, 38-42. Moscow: Nauka.

5. Savitsky, E.M., Burkhanov, G.S., Raskatov, N.N. (1978) Application of plasma heating for growing the single crystals of refractory metals. In: Metal single crystals, 5-10. Moscow: Nauka.

6. Shapovalov, V., Yakusha, V., Manulyk, A. (2015) Large refractory metals single crystals grown by plasma-induction zone melting. In: Proc. of $24^{\text {th }}$ Int. Materials Research Congress (Cancun, Mexico, 16-20 Aug., 2015).

7. Shapovalov, V.A., Yakusha, V.V., Nikitenko, Yu.A. (2014) Investigation of temperature field of profiled tungsten single crystals made by plasma-induction method. Sovr. Elektrometallurgiya, 3, 31-35.

8. Smirnov, V.P. (2008) Thermonuclear power as the major international innovative project. Ros. Khimich. Zhurnal, 6, 79-94.

9. Filatov, O.G., Mazul, I.V. (2003) NII EFA experimental complex for simulation of operational factor of first wall of ITER reactor. Voprosy Atomn. Nauki i Tekhniki, 3, 3-31. 